Pholani Manana, Eric C. Hosten and Richard Betz*

\title{
Crystal structure of benzanthrone - a redetermination for correct molecular geometry and localization of hydrogen atoms
}

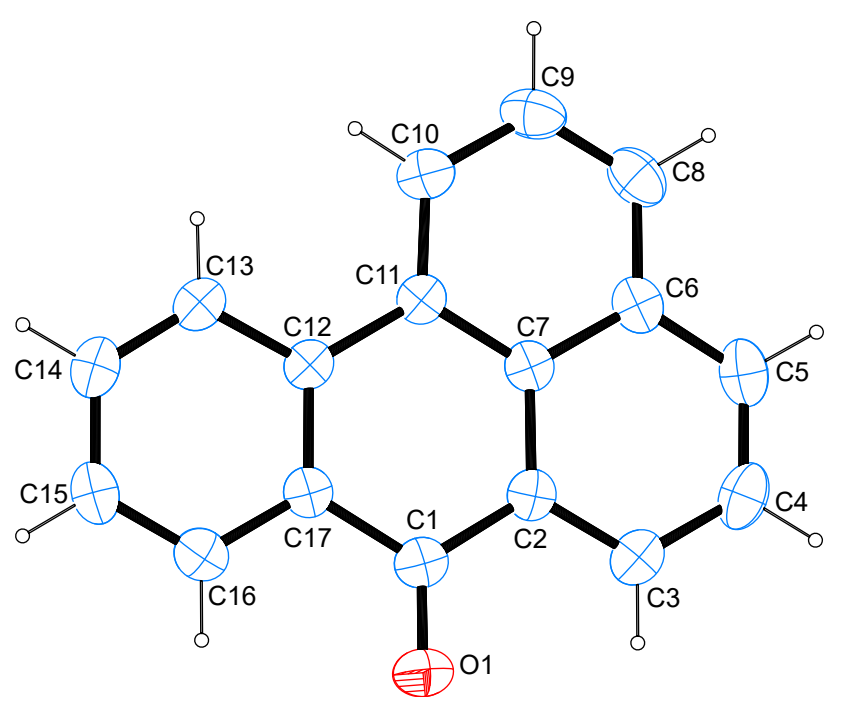

https://doi.org/10.1515/ncrs-2020-0389

Received July 23, 2020; accepted September 8, 2020;

published online October 12, 2020

\section{Abstract}

$\mathrm{C}_{17} \mathrm{H}_{10} \mathrm{O}$, orthorhombic, $P 2_{1} 2_{1} 2_{1}$ (no. 19), $a=5.0604(12) \AA$, $b=14.672(3) \AA, c=14.846(3) \AA, V=1102.2(4) \AA^{3}, Z=4$, $R_{g t}(F)=0.0432, w R_{\text {ref }}\left(F^{2}\right)=0.1022, T=200(2) \mathrm{K}$.

\section{CCDC no.: 2027362}

The molecular structure is shown in the figure. Table 1 contains crystallographic data and Table 2 contains the list of the atoms including atomic coordinates and displacement parameters.

*Corresponding author: Richard Betz, Department of Chemistry, Nelson Mandela University, Summerstrand Campus (South) University Way, Summerstrand, PO Box 77000, Port Elizabeth, 6031, South Africa, e-mail: Richard.Betz@mandela.ac.za.https://orcid.org/00000002-5730-2152

Pholani Manana and Eric C. Hosten, Department of Chemistry, Nelson Mandela University, Summerstrand Campus (South) University Way, Summerstrand, PO Box 77000, Port Elizabeth, 6031, South Africa. https://orcid.org/0000-0003-4173-2550 (E.C. Hosten)
Table 1: Data collection and handling.

\begin{tabular}{ll}
\hline Crystal: & Yellow needles \\
Size: & $0.45 \times 0.09 \times 0.05 \mathrm{~mm}$ \\
Wavelength: & Mo $K \alpha$ radiation $(0.71073 \AA$ A $)$ \\
$\mu:$ & $0.09 \mathrm{~mm}^{-1}$ \\
Diffractometer, scan mode & Bruker APEX-II, $\varphi$ and $\omega$ \\
$\theta_{\text {max }}$, completeness: & $28.4^{\circ},>99 \%$ \\
$N(h k l)_{\text {measured }}$, & $8990,1620,0.034$ \\
$\quad N(h k l)_{\text {unique }}, R_{\text {int }}:$ & \\
Criterion for $I_{\text {obs }}, N(h k l)_{\text {gt }}:$ & $I_{\text {obs }}>2 \sigma\left(I_{\text {obs }}\right), 1181$ \\
$N(\text { param })_{\text {refined }}:$ & 163 \\
Programs: & Bruker [1], SHELX [2], WinGX/ORTEP [3], \\
& Mercury [4], PLATON [5] \\
\hline
\end{tabular}

Table 2: Fractional atomic coordinates and isotropic or equivalent isotropic displacement parameters $\left(\AA^{2}\right)$.

\begin{tabular}{lrrrr}
\hline Atom & $\boldsymbol{x}$ & $\boldsymbol{y}$ & $\boldsymbol{z}$ & \multicolumn{1}{c}{$\boldsymbol{U}_{\text {iso }} / \boldsymbol{U}_{\text {eq }}$} \\
\hline O1 & $0.4104(4)$ & $0.61016(11)$ & $0.42422(10)$ & $0.0477(5)$ \\
C1 & $0.4282(5)$ & $0.56471(15)$ & $0.49358(14)$ & $0.0319(5)$ \\
C2 & $0.6197(5)$ & $0.48920(14)$ & $0.50153(13)$ & $0.0289(5)$ \\
C3 & $0.7967(5)$ & $0.47345(16)$ & $0.43227(14)$ & $0.0347(5)$ \\
H3 & 0.7934 & 0.5114 & 0.3804 & $0.042^{*}$ \\
C4 & $0.9805(5)$ & $0.40242(17)$ & $0.43752(15)$ & $0.0398(6)$ \\
H4 & 1.1005 & 0.3923 & 0.3893 & $0.048^{*}$ \\
C5 & $0.9877(5)$ & $0.34754(16)$ & $0.51192(15)$ & $0.0388(6)$ \\
H5 & 1.1134 & 0.2995 & 0.5149 & $0.047^{*}$ \\
C6 & $0.8109(5)$ & $0.36103(16)$ & $0.58468(14)$ & $0.0319(5)$ \\
C7 & $0.6232(4)$ & $0.43306(15)$ & $0.58005(13)$ & $0.0269(5)$ \\
C8 & $0.8182(5)$ & $0.30526(17)$ & $0.66258(16)$ & $0.0414(6)$ \\
H8 & 0.9441 & 0.2574 & 0.6667 & $0.050^{*}$ \\
C9 & $0.6457(5)$ & $0.31979(18)$ & $0.73170(16)$ & $0.0436(6)$ \\
H9 & 0.6518 & 0.2817 & 0.7834 & $0.052^{*}$ \\
C10 & $0.4588(5)$ & $0.39063(16)$ & $0.72723(14)$ & $0.0353(6)$ \\
H10 & 0.3397 & 0.3994 & 0.7759 & $0.042^{*}$ \\
C11 & $0.4449(4)$ & $0.44801(14)$ & $0.65308(13)$ & $0.0274(5)$ \\
C12 & $0.2575(4)$ & $0.52501(14)$ & $0.64842(13)$ & $0.0271(5)$ \\
C13 & $0.0774(5)$ & $0.54382(16)$ & $0.71803(13)$ & $0.0326(5)$ \\
H13 & 0.0743 & 0.5059 & 0.7699 & $0.039^{*}$ \\
C14 & $-0.0946(5)$ & $0.61629(16)$ & $0.71222(14)$ & $0.0363(6)$ \\
H14 & -0.2161 & 0.6272 & 0.7598 & $0.044^{*}$ \\
C15 & $-0.0930(5)$ & $0.67382(16)$ & $0.63775(15)$ & $0.0380(6)$ \\
H15 & -0.2099 & 0.7244 & 0.6349 & $0.046^{*}$ \\
C16 & $0.0800(5)$ & $0.65659(15)$ & $0.56824(15)$ & $0.0361(5)$ \\
H16 & 0.0818 & 0.6955 & 0.5171 & $0.043^{*}$ \\
C17 & $0.2529(5)$ & $0.58257(14)$ & $0.57199(13)$ & $0.0287(5)$ \\
\hline & & & &
\end{tabular}




\section{Source of material}

The compound was obtained commercially (Aldrich). Crystals suitable for the X-ray diffration study were obtained upon very slow cooling of the compound's solution in toluene in a hot-water bath overnight.

\section{Experimental details}

Benzene and its higher-annealed aromatic derivatives are among the most important synthons in chemistry. Via electrophilic substitution reactions a vast variety of functionalized derivatives is readily available. The interplay between activating and deactivating substituents as well as the competition and synergism between inductive and mesomeric effects allows for the seemingly endless functionalization of the respective archaetype hydrocarbon scaffold. The latter gives rise to a large toolbox of new synthons that can be applied for the production of dyes, medications, catalysts and ligands for novel coordination compounds. Especially hydroxycarboxylic acids that can easily be derived from cyanohydrines [6] are interesting in this aspect, as the extended aromatic systems are prone to improve the crystallizability of pertaining reaction products, which allows for a determination and unambiguous identification of bonding patterns and metrical properties in envisioned target molecules based on single-crystal diffraction studies. An interesting starting point was seen in the benzoanthracene scaffold as it offers four annealed phenyl moieties and a pertaining ketone is readily available. To account for the effect that the formation of the hydroxycarboxylic acid as well as its coordination to transition metals will have on the metric parameters within the molecule, the structure of the title compound seemed of interest as well. An initial search of the Cambridge Structural Database [7] showed that the molecular and crystal structure of the title compound been deposited and reported twice earlier, however, one entry (ref code: ZZZUBM) [8] does not feature 3D data deposited while the atomic coordinates deposited for the second entry (ref code: SIHCIV) [9] are dubious as they show the molecule bent in the middle, which contradicts all expectation for a fully $\mathrm{sp}^{2}$ hybridized system. Furthermore, this CSD entry lacks two hydrogen atoms entirely while the remaining ones assume improbable orientations and $\mathrm{C}-\mathrm{H}$ bond lengths. In this context it is pertinent to point out that the notes in the database explicitly state for this set of data that the two missing hydrogen atoms had been removed due to suspected erroneous coordinates. As the absence of reliable data precludes comparative follow-up investigations from a metrical point of view as of this moment, this study is intended to close this gap.

The title compound is a ketone derived from benzoanthracene, a benzo-annealed derivative of anthracene. The annealing is effected via two peri-positioned hydrogen atoms, i.e. the additional six-membered ring shares two sides with the anthracene scaffold. Unlike the 3D data mentioned earlier [9], the molecule is found to be essentially flat - the least-squares plane as defined by all nonhydrogen atoms within the molecule shows the largest deviation from this place for the exocyclic oxygen atom with a value of $-0.117(2) \AA$. The largest deviation for a carbon atom in the same context was measured at only $0.068(2) \AA$ A. The least-squares planes as defined by the carbon atoms of each individual six-membered ring enclose angles of 1.51(10)-1.60(10) ${ }^{\circ}$ for the oxygen-bearing ring while the annealed six-membered ring along the linear extension of the anthracene scaffold intersects at an angle of $0.28(11)^{\circ}$ with the two-fold annealing phenyl moiety and of $2.51(11)^{\circ}$ towards the ring bearing the highest number of hydrogen atoms. The largest angle of intersection is found for the least-squares planes as defined by the carbon atoms of the latter ring and of the annealing phenyl moiety that was added to the anthracene scaffold. The $\mathrm{C}=\mathrm{O}$ bond length was found at 1.230(2) $\AA$ and does not deviate significantly from other ketones whose metrical parameters have been deposited with the Cambridge Structural Database.

In the crystal, $\mathrm{C}-\mathrm{H} \cdots \mathrm{O}$ contacts, whose range falls by more than 0.1 A below the sum of van-der-Waals radii of the atoms participating in them, are apparent. These are supported by the hydrogen atom on the carbon atom in the "extended" para position to the keto group. In terms of graphset-analysis [10,11], the descriptor for these contacts is $C_{1}^{1}(7)$ at the primary level. Overall, these contacts connect the molecules to form undulated chains along the crystallographic $b$ axis. Significant $\pi$ stacking is observed in the crystal structure with the shortest distance between two centers of gravity measured at 3.6074(16) A. This interaction is observed between the two six-membered rings of the linear anthracene scaffold that bear the two-fold annealed phenyl moiety.

Acknowledgments: The authors thank Mr Henk Schalekamp for helpful discussions.

Author contribution: All the authors have accepted responsibility for the entire content of this submitted manuscript and approved submission.

Research funding: None declared.

Conflict of interest statement: The authors declare no conflicts of interest regarding this article. 


\section{References}

1. Bruker. SAINT, APEX2 and SADABS; Bruker AXS Inc.: Madison, Wisconsin, USA, 2009.

2. Sheldrick G. M. Crystal structure refinement with SHELXL. Acta Crystallogr. 2015, C71, 3-8.

3. Farrugia L. J. WinGX and ORTEP for windows: an update. J. Appl. Crystallogr. 2012, 45, 849-854.

4. Macrae C. F., Bruno I. J., Chisholm J. A., Edgington P. R., McCabe P., Pidcock E., Rodriguez-Monge L., Taylor R., van de Streek J., Wood P. A. Mercury CSD 2.0 - new features for the visualization and investigation of crystal structures. J. Appl. Crystallogr. 2008, 41, 466-470.

5. Spek A. L. Structure validation in chemical crystallography. Acta Crystallogr. 2009, D65, 148-155.

6. Becker H. G. O., Beckert R., Domschke G., Fanghänel E., Habicher W. D., Metz P., Pavel D., Schwetlick K. Organikum - Organisch- chemisches Grundpraktikum, 21st ed.; Wiley-VCH: Weinheim, 2001.

7. Allen F. H. The Cambridge structural database: a quarter of a million crystal structures and rising. Acta Crystallogr. 2002, B58, 380-388.

8. Boyd H. C., Edward P. B. M., Goodwin T. H., Hicks D., MacFarlane J., MacNaughton L. Cell dimensions and space groups of some carbocyclic compounds. Acta Crystallogr. 1954, 7, 142.

9. Tafeenko V. A., Solodar' S. L., Medvedev S. V. X-ray structural study of 2-acetylaminophenalenone and benzanthrone. Russ. J. Gen. Chem. 1988, 58, 2600-2605.

10. Bernstein J., Davis R. E., Shimoni L., Chang N.-L. Patterns in hydrogen bonding: functionality and graph set analysis in crystals. Angew. Chem. Int. Ed. Engl. 1995, 34, 1555-1573.

11. Etter M. C., MacDonald J. C., Bernstein J. Graph-set analysis of hydrogen-bond patterns in organic crystals. Acta Crystallogr. 1990, B46, 256-262. 